\title{
Position Vectors of General Helices According to Type-2 Bishop Frame in $E^{3}$
}

\author{
Hülya Gün Bozok*, Sezin Aykurt Sepet and Mahmut Ergüt \\ (Communicated by Ákos G. HORVÁTH)
}

\begin{abstract}
In this paper, we study the position vector of a general helix according to type-2 Bishop frame in the 3-dimensional Euclidean space $E^{3}$. Moreover we determine the natural representation of a general helix in $E^{3}$.
\end{abstract}

Keywords: Position vector; type-2 Bishop frame.

AMS Subject Classification (2010): 53A04.

${ }^{*}$ Corresponding author

\section{Introduction}

The theory of curves has an important role in differential geometry. One of the most interesting curve used in nature and science is helix. A helix is a curve with non-vanishing curvature and torsion. This fascinating curve arises in nature such that DNA double, carbon nano-tubes, nano springs, etc.[6, 8]. Intrinsically a helix also known a circular helix or W-curve is a special case of general helix [5, 7]. A general helix or a curve of constant slope is determined that the tangent makes a constant angle with a fixed straight line called the axis of general helix [3]. An important result for general helix stated by Lancret (1802) and proved by de Saint Venant (1945) is: a necessary and sufficient condition that a curve be general helix is that ratio of curvature to torsion be constant [11] .

On the other hand, L.R. Bishop defined Bishop frame, which is known alternative or parallel frame of the curves with the help of parallel vector fields [4]. Then, S. Yilmaz and M. Turgut introduced a new version of the Bishop frame which is called type-2 Bishop frame [12]. Thereafter, E. Ozyilmaz studied classical differential geometry of curves according to type-2 Bishop trihedra [9].

Position vectors of curves investigated by many researchers, for example in [1] and [2] position vectors of general and slant helices in 3-dimensional Euclidean space are researched, in [7] position vector of a spacelike W-curve in Minkowski space is obtained and in [10] position vectors of admissible curves in 3-dimensional pseudo-Galilean space are determined.

In this study we research position vectors of a general helix according to type-2 Bishop frame in the 3-dimensional Euclidean space $E^{3}$, and we obtain the natural representation of a general helix.

\section{Preliminaries}

The standard flat metric of 3-dimensional Euclidean space $E^{3}$ is given by

$$
\langle,\rangle: d x_{1}^{2}+d x_{2}^{2}+d x_{3}^{2}
$$

where $\left(x_{1}, x_{2}, x_{3}\right)$ is a rectangular coordinate system of $E^{3}$. For an arbitrary vector $x$ in $E^{3}$, the norm of this vector is defined by $\|x\|=\sqrt{\langle x, x\rangle}$. $\alpha$ is called a unit speed curve, if $\left\langle\alpha^{\prime}, \alpha^{\prime}\right\rangle=1$. Suppose that $\{t, n, b\}$ is the moving

Received : 14-03-2018, Accepted : 19-04-2018 
Frenet-Serret frame along the curve $\alpha$ in $E^{3}$. For a unit speed curve $\alpha$, the Frenet-Serret formulae can be given as

$$
\begin{aligned}
t^{\prime} & =\kappa n \\
n^{\prime} & =-\kappa t+\tau b \\
b^{\prime} & =-\tau n
\end{aligned}
$$

where

$$
\begin{aligned}
& \langle t, t\rangle=\langle n, n\rangle=\langle b, b\rangle=1, \\
& \langle t, n\rangle=\langle t, b\rangle=\langle n, b\rangle=0 .
\end{aligned}
$$

and here, $\kappa=\kappa(s)=\left\|t^{\prime}(s)\right\|$ and $\tau=\tau(s)=-\left\langle n, b^{\prime}\right\rangle$. Furthermore, the torsion of the curve $\alpha$ can be given

$$
\tau=\frac{\left[\alpha^{\prime}, \alpha^{\prime \prime}, \alpha^{\prime \prime \prime}\right]}{\kappa^{2}}
$$

Along the paper, we assume that $\kappa \neq 0$ and $\tau \neq 0$.

Bishop frame is an alternative approachment to define a moving frame. Assume that $\alpha(s)$ is a unit speed regular curve in $E^{3}$. The type-2 Bishop frame of the $\alpha(s)$ is expressed as [12]

$$
\begin{aligned}
N_{1}^{\prime} & =-k_{1} B, \\
N_{2}^{\prime} & =-k_{2} B, \\
B^{\prime} & =k_{1} N_{1}+k_{2} N_{2} .
\end{aligned}
$$

The relation matrix may be expressed as

$$
\left[\begin{array}{l}
t \\
n \\
b
\end{array}\right]=\left[\begin{array}{ccc}
\sin \theta(s) & -\cos \theta(s) & 0 \\
\cos \theta(s) & \sin \theta(s) & 0 \\
0 & 0 & 1
\end{array}\right]\left[\begin{array}{l}
N_{1} \\
N_{2} \\
B
\end{array}\right] .
$$

where $\theta(s)=\int_{0}^{s} \kappa(s) d s$. Then, type-2 Bishop curvatures can be defined in the following

$$
\begin{gathered}
k_{1}(s)=-\tau(s) \cos \theta(s), \\
k_{2}(s)=-\tau(s) \sin \theta(s) .
\end{gathered}
$$

On the other hand,

$$
\theta^{\prime}=\kappa=\frac{\left(\frac{k_{2}}{k_{1}}\right)^{\prime}}{1+\left(\frac{k_{2}}{k_{1}}\right)^{2}} .
$$

The frame $\left\{N_{1}, N_{2}, B\right\}$ is properly oriented, $\tau$ and $\theta(s)=\int_{0}^{s} \kappa(s) d s$ are polar coordinates for the curve $\alpha$. Then, $\left\{N_{1}, N_{2}, B\right\}$ is called type-2 Bishop trihedra and $k_{1}, k_{2}$ are called Bishop curvatures.

\section{Position Vectors of a General Helix According to type-2 Bishop Frame}

Theorem 3.1. Let $\alpha(x)$ be a general helix with $k_{1}(x) \neq 0$. Then its position vector with respect to type-2 Bishop frame is given by

$$
\alpha(x)=\lambda N_{1}+\mu N_{2}+\gamma B
$$


where

$$
\begin{aligned}
\lambda= & \int \frac{1}{k_{1}(x)} d x \\
- & \int\left[\left(\frac{1}{\sqrt{1+m^{2}}} \int \cos \left(\sqrt{1+m^{2}} \int k_{1}(x) d x\right) d x+c_{1}\right)\right. \\
& \left.\sin \left(\sqrt{1+m^{2}} \int k_{1}(x) d x\right)\right] d x \\
+ & \int\left[\left(\frac{1}{\sqrt{1+m^{2}}} \int \sin \left(\sqrt{1+m^{2}} \int k_{1}(x) d x\right) d x+c_{2}\right)\right. \\
\mu & \left.\cos \left(\sqrt{1+m^{2}} \int k_{1}(x)\right) d x\right] d x \\
= & -m \int\left[\left(\frac{1}{\sqrt{1+m^{2}}} \int \cos \left(\sqrt{1+m^{2}} \int k_{1}(x) d x\right) d x+c_{1}\right)\right. \\
& \left.\sin \left(\sqrt{1+m^{2}} \int k_{1}(x) d x\right)\right] d x \\
+ & m \int\left[\left(\frac{1}{\sqrt{1+m^{2}}} \int \sin \left(\sqrt{1+m^{2}} \int k_{1}(x) d x\right) d x+c_{2}\right)\right. \\
\gamma= & \left.\cos \left(\sqrt{1+m^{2}} \int k_{1}(x) d x\right)\right] d x \\
\gamma & {\left[\frac{1}{\sqrt{1+m^{2}}} \int \cos \left(\sqrt{1+m^{2}} \int k_{1}(x) d x\right) d x+c_{1}\right] \sin \left(\sqrt{1+m^{2}} \int k_{1}(x) d x\right) } \\
- & {\left[\frac{1}{\sqrt{1+m^{2}}} \int \sin \left(\sqrt{1+m^{2}} \int k_{1}(x) d x\right) d x+c_{2}\right] \cos \left(\sqrt{1+m^{2}} \int k_{1}(x) d x\right) }
\end{aligned}
$$

and where $\frac{k_{2}(x)}{k_{1}(x)}=m=$ constant.

Proof. Let $\alpha(x)$ be a general helix with $k_{1}(x) \neq 0$ in $E^{3}$. If $\lambda, \mu, \gamma$ are differentiable functions of $x \in I \subset \mathbb{R}$ then its position vectors according to type-2 Bishop frame can be written

$$
\alpha(x)=\lambda(x) N_{1}(x)+\mu(x) N_{2}(x)+\gamma(x) B(x) .
$$

If $N_{1}$ is taken instead of tangent vector, differentiating above equation according to $x$ and considering type-2 Bishop frame we have the following

$$
\begin{aligned}
\lambda^{\prime}+\gamma k_{1}-1 & =0 \\
\mu^{\prime}+\gamma k_{2} & =0 \\
\gamma^{\prime}-\lambda k_{1}-\mu k_{2} & =0 .
\end{aligned}
$$

If we can change the variable $x$ by the variable $\theta=\int k_{1}(x) d x$, then all functions of $x$ will turn into the functions of $\theta$ (such that $\lambda(\theta)=(\lambda \circ x)(\theta)$ ). Therefore the equation (3.3) is written as

$$
\begin{aligned}
\dot{\lambda}+\gamma-\frac{1}{k_{1}} & =0 \\
\dot{\mu}+\gamma \frac{k_{2}}{k_{1}} & =0 \\
\dot{\gamma}-\lambda-\mu \frac{k_{2}}{k_{1}} & =0
\end{aligned}
$$

where the dot denote the derivative according to $\theta$. Then differentiating equation (3.6) and substiuting the equations (3.4) and (3.5) in the equation (3.6) we get the following differential equation

$$
\ddot{\gamma}+\left(1+\frac{k_{2}^{2}}{k_{1}^{2}}\right) \gamma-\frac{1}{k_{1}}=0
$$

If we solve above equation and setting $\theta=\int k_{1}(x) d x$ we obtain the equation (3.1) which complete the proof. 
Lemma 3.1. Let $\alpha(x)$ be a circular helix, then its position vector with respect to type-2 Bishop frame is given by

$$
\alpha(x)=\lambda N_{1}+\mu N_{2}+\gamma B
$$

where

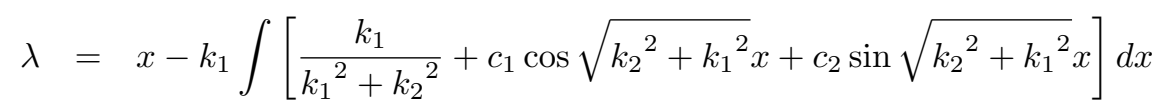

$$
\begin{aligned}
& \mu=-\frac{k_{2}}{k_{1}} \int\left[\frac{k_{1}}{{k_{1}{ }^{2}+k_{2}^{2}}^{2}}+c_{1} \cos \sqrt{{k_{2}}^{2}+{k_{1}}^{2}} x+c_{2} \sin \sqrt{{k_{2}}^{2}+k_{1}^{2}} x\right] d x
\end{aligned}
$$

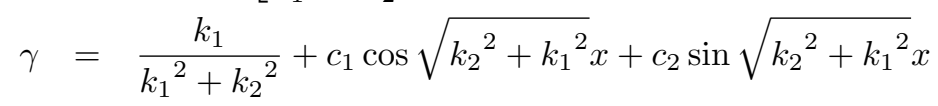

\section{Position Vectors of a General Helix with Respect to Standart Frame}

Theorem 4.1. Let $\alpha(x)$ be a general helix with $k_{1}(x) \neq 0$, then its tangent vector makes a constant angle with a fixed straight line in space. So, $\alpha(x)$ is determined in the natural representation form in the following

$$
\begin{aligned}
\alpha(x)= & \int\left[\int \left(-k_{1}(x) \cos \left(1+\frac{k_{2}^{2}(x)}{k_{1}(x)}\right) \int k_{1}(x) d x,\right.\right. \\
& \left.\left.-k_{1}(x) \sin \left(1+\frac{k_{2}^{2}(x)}{k_{1}(x)}\right) \int k_{1}(x) d x, 0\right) d x\right] d x
\end{aligned}
$$

Proof. Let $\alpha(x)$ be a general helix. Using the third equation of (2.3) we obtain

$$
N_{1}(x)=\left(\frac{1}{k_{1}(x)}\right) B^{\prime}(x)-\left(\frac{k_{2}(x)}{k_{1}(x)}\right) N_{2}(x)
$$

Moreover the first equation of (2.3) we have

$$
N_{1}^{\prime}(x)+k_{1}(x) B^{\prime}(x)=0
$$

If we can change the variable $x$ by the variable $\theta=\int k_{1}(x) d x$. So, all functions of $x$ will turn into the functions of $\theta$. Then differentiating the equation (4.2) and using the equation (4.3) we get the following differential equation

$$
\ddot{B}+\left(1+\frac{k_{2}^{2}}{k_{1}}\right) B=0
$$

where the dot denote the derivative according to $\theta$. If $N_{1}$ is taken instead of tangent vector and $\alpha$ be a general helix, then $N_{1}$ makes a constant angle $\phi$ with the constant vector called the axis of helix. So, without loss of generality, we take the axis to be parallel to $e_{3}$. Then,

$$
N_{1}=\left(N_{1}\right)_{1}(\theta) e_{1}+\left(N_{1}\right)_{2}(\theta) e_{2}+\left(N_{1}\right)_{3}(\theta) e_{3}
$$

and

$$
\left(N_{1}\right)_{3}(\theta)=\left\langle N_{1}, e_{3}\right\rangle=\cos (\phi)=n
$$

Also we have

$$
B=B_{1}(\theta) e_{1}+B_{2}(\theta) e_{2}+B_{3}(\theta) e_{3}
$$

and differentiating the equation (4.5) we get

$$
B_{3}(\theta)=\left\langle B, e_{3}\right\rangle=0
$$

Because of $B$ is a unit vector, then the condition that must be satisfied is as follows

$$
B_{1}^{2}(\theta)+B_{2}^{2}(\theta)=1
$$


The general solution of this equation can be given by

$$
B_{1}=\cos [t(\theta)], \quad B_{2}=\sin [t(\theta)],
$$

where $t$ is an arbitrary function of $\theta$. So, substiuting the components of $B$ in the equation (4.4) we obtain

$$
\begin{aligned}
-\ddot{t} \sin (t)+\left[1+\left(\frac{k_{2}{ }^{2}}{k_{1}}\right)-\dot{t}\right] \cos (t) & =0 \\
\ddot{t} \cos (t)+\left[1+\left(\frac{k_{2}^{2}}{k_{1}}\right)-\dot{t}\right] \sin (t) & =0 .
\end{aligned}
$$

The above equations can be reduced in the following

$$
\begin{aligned}
\ddot{t} & =0 \\
{\left[1+\left(\frac{k_{2}^{2}}{k_{1}}\right)\right]-\dot{t} } & =0
\end{aligned}
$$

The general solution of equation (4.9) is

$$
t(\theta)=c_{1} \theta+c_{2}
$$

where $c_{1}$ and $c_{2}$ are constants. Using the equation (4.11) in (4.10) we obtain

$$
c_{1}=\left[1+\left(\frac{k_{2}^{2}}{k_{1}}\right)\right]
$$

So, we can write

$$
t(\theta)=\left[1+\left(\frac{k_{2}^{2}}{k_{1}}\right)\right] \theta+c_{2}
$$

The constants of $c_{2}$ are disappear when making a change such that $t \rightarrow t+c_{2}$. Therefore the unit normal vector $B$ is find as follows

$$
B=\left(\cos \left[1+\left(\frac{k_{2}^{2}}{k_{1}}\right)\right] \int k_{1}(x) d x, \sin \left[1+\left(\frac{k_{2}^{2}}{k_{1}}\right)\right] \int k_{1}(x) d x, 0\right)
$$

Considering the equation (2.3) and (4.12) we obtain the equation (4.1) which complete the proof.

Lemma 4.1. Let $\alpha(x)$ be a circular helix, So, $\alpha(x)$ is determined in the natural representation form in the following

$$
\alpha(x)=\int\left[-k_{1} \int\left(\cos \left(k_{1}^{2}+k_{2}^{2}\right) x, \sin \left(k_{1}^{2}+k_{2}^{2}\right) x, 0\right) d x\right] d x
$$

\section{References}

[1] Ahmad, T.A., Position vectors of general helices in Euclidean 3-Space, Bull. Math. Anal. Appl., 3 (2011) no. 2, 198-205.

[2] Ahmad, T.A., Position vectors of slant helices in Euclidean 3-space, J. Egyp. Math. Soc., 20 (2012), 1-6.

[3] Barros, M., General helices and a theorem of Lancret, Proc. Am. Math. Soc., 125 (1997), 1503-1509.

[4] Bishop, L.R., There is more than one way to frame a curve, Am. Math. Monthly, 82 (1975) no. 3, 246-251.

[5] Chen, B.Y., Kim D.S., Kim Y.H., New characterizations of W-curves, Publ. Math. Debrecen, 69 (2006), 457-472.

[6] Chouaieb, N., Goriely, A., Maddocks, J.H. , Helices, PANS, 103 (2006), 9398-9403.

[7] Ilarslan, K., Boyacioglu, O., Position vectors of a space like W-curve in Minkowski space $E_{1}^{3}$, Bull. Korean Math. Soc., 44 (2007), 429-438.

[8] Lucas, A.A., Lambin, P., Diffraction by DNA, carbon nanotubes and other helical nanostructures, Rep. Prog. Phys., 68, 1181-1249, 2005. 
[9] Ozyilmaz, E., Classical differential geometry of curves according to type-2 bishop trihedra, Math. Comput. Appl., 16 (2011) no. 4, 858-867.

[10] Oztekin, H. and Gun Bozok, H., Position vectors of admissible curves in 3-dimensional pseudo-Galilean space $G_{3}^{1}$, Int. Electron. J. Geom., 8 (2015) no. 1, 21-32.

[11] Struik, D.J., Lectures in Classical Differential Geometry, Addison,-Wesley, Reading, MA, 1961.

[12] Yilmaz, S. and Turgut, M., A new version of Bishop frame and an application to spherical images, J. Math. Anal. Appl., 371 (2010), 764-776.

\section{Affiliations}

HÜLYA GÜN BOZOK

AdDRESS: Osmaniye Korkut Ata University, Dept. of Mathematics, 80000, Osmaniye-Turkey.

E-MAIL: hulyagun@osmaniye.edu.tr

ORCID ID: $0000-0002-7370-5760$

SEZIN AYKURT SEPET

AdDress: Ahi Evran University, Dept. of Mathematics, 40200, Kirsehir-Turkey.

E-MAIL: sezinaykurt@hotmail.com

ORCID ID: $0000-0003-1521-6798$

MAHMUT ERGÜT

AdDress: Namik Kemal University, Dept. of Mathematics, 59000, Tekirdag-Turkey.

E-MAIL: mergut@nku.edu.tr

ORCID ID: $0000-0002-9098-8280$ 